\title{
The Development and Validation of a Prognostic Nomogram and Nomogram Application Software Among Men Treated with Abiraterone Acetate and/or Enzalutamide for Metastatic Castration- resistant Prostate Cancer
}

Takashi Kawahara ( $\square$ takashi_tk2001@yahoo.co.jp)

Yokohama City University Medical Center https://orcid.org/0000-0002-7049-3379

Yusuke Saigusa

Yokohama City University School of Medicine

Shuko Yoneyama

Yokohama City University Medical Center

Masashi Kato

Nagoya University

Ippei Kojima

Nagoya University

Hiroshi Yamada

Nagoya University

Osamu Kamihira

Nagoya University

Kenichi Tabata

Kitasato University

Hideyasu Tsumura

Kitasato University

Masatsugu Iwamura

Kitasato University

Masahiro Yao

Yokohama City University School of Medicine

Hiroji Uemura

Yokohama City University Medical Center

Yasuhide Miyoshi

Yokohama City University Medical Center 
Keywords: CRPC, prognosis, Enzalutamide, Abiraterone acetate, nomogram

Posted Date: August 26th, 2020

DOI: https://doi.org/10.21203/rs.3.rs-58034/v1

License: (c) (i) This work is licensed under a Creative Commons Attribution 4.0 International License. Read Full License 


\section{Abstract}

\section{Background:}

With widespread medication choices for metastatic castration-resistant prostate cancer (mCRPC) is now available, on the other hand biomarker to predict the efficacy of each mCRPC treatment has not been established.

\section{Objective:}

This study developed prognostic nomogram to predict prognosis in CRPC patients who received abiraterone acetate $(A B I)$ and/or enzalutamide (ENZ).

\section{Design, Setting, and Participants:}

A total of 568 mCRPC patients received ABI and/or ENZ from 2012 to 2017 were enrolled in this study. We developed prognostic nomogram based on the risk factors by Cox proportional hazards regression model.

\section{Outcome Measurements and Statistical Analysis:}

The nomogram was also assessed for discriminatory ability with the concordance index (C-index). We repeated 5 -fold cross-validation 2000 times to estimate the C-index and reported the means of the estimated C-index for the training and validation sets. And we also developed nomogram application software (app) based on this nomogram.

\section{Results and Limitations:}

The median overall survival (OS) was 24.7 months. A multivariable analysis showed that the time to CRPC, pre-chemotherapy, baseline PSA, baseline ALP, and baseline LDH were independent risk factors for the OS (HR: $0.521,1.681,1.439,1.827,12,123, \mathrm{p}: 0.001,0.001,<0.001,0.019,<0.001)$ ). C-index was 0.72 in training cohort and 0.71 in validation cohort.

\section{Conclusion:}

We developed nomograms to predict the OS for Japanese MCRPC patients who received ABI and/or ENZ. The advent of $\mathrm{mCRPC}$ prognosis prediction app will facilitate greater accessibility for clinical use.

\section{Patient Summary}

This study developed and validated a nomogram for predicting the prognosis of mCRPC patients who receive $A B I / E N Z$ treatment using clinical information. This study also developed mobile app to facilitate clinical usage. 


\section{Introduction}

A recent clinical trial revealed the efficacy of abiraterone acetate ( $A B I)$, enzalutamide (ENZ), Radium-223 (Ra-223), and cabazitaxel in addition to docetaxel chemotherapy in metastatic castration-resistant prostate cancer (mCPRC) patients [1-3]. In the next few years, poly(ADP-ribose) polymerase (PARP) inhibitors and immune-checkpoint inhibitors are expected to be used in clinical practice to similar ends [4, 5]. With widespread medication choices now available, clinicians should take care to select the most appropriate medicine in order not to lose their chance to administer the best therapy possible to a patient. Predicting the prognosis is thus important, because a lack of biomarker to predict the efficacy of each mCRPC treatment.

Recent studies have demonstrated the efficacy of tumor markers for predicting the prognosis, such as inflammatory markers, including the neutrophil-to-lymphocyte ratio, monocyte-to-lymphocyte ration, and platelet-to-lymphocyte ratio, as well as the alkaline phosphatase (ALP) and lactate dehydrogenase (LDH) levels [6]. However, to more accurately predict the prognosis, a nomogram using multiple prognostic parameters is needed $[7,8]$.

In the present study, we developed and validated a nomogram for predicting the prognosis of mCRPC patients who receive $\mathrm{ABI}$ and/or ENZ treatment using clinical information and also developed application software (app) to facilitate clinical usage.

\section{Materials And Methods}

\section{Patients}

A total of 568 metastatic CRPC (mCRPC) patients received ABI and/or ENZ in Yokohama City University, Nagoya University, Kitasato University, and affiliated hospitals from 2012 to 2017 . All cases were pathologically confirmed to have prostate cancer and received androgen deprivation therapy (ADT) but proved refractory.

The institutional review board of Yokohama City University Medical Center was approved this study (D1603004). The definition of CRPC was set by the Prostate Cancer Working Group 2 [9]. Patients' background characteristics, including the initial prostate-specific antigen (PSA) level, initial metastatic status, Gleason score, observation period, time to CRPC, pre-chemotherapy, age at baseline, PSA at baseline, ALP at baseline, LDH as baseline, and initial ABI/ENZ treatment, are shown in Table 1. 
Table 1

Patients' background

\section{Variables}

Initial PSA, ng/mL

Initial stage

M0

M1

Gleason score

6,7

$8-10$

Observation period, months

Time to CRPC, months

Previous use of chemotherapy

Age at baseline, yeas

PSA at baseline, $\mathrm{ng} / \mathrm{mL}$

ALP at baseline, IU/L

LDH at baseline, IU/L

Treatment

$A B I$

ENZ n (\%), median (range)

123.0(2.1-19840.0)

$215(37.9 \%)$

$353(62.1 \%)$
$128(22.5 \%)$

440 (77.5\%)

$13.3(0.2-52.2)$

$14.2(0.4-189.1)$

$202(35.6 \%)$

76 (47-92)

$23.6(0.1-10,000)$

$261(60-6,908)$

$215(93-3201)$

$234(41.2 \%)$

$334(58.8 \%)$

PSA: Prostate-specific antigen, ALP: alkaline phosphatase, LDH: lactate dehydrogenase, CRPC:

Castration-resistant prostate cancer,

ABI: Abiraterone acetate, ENZ: Enzalutamide

\section{Statistical analyses}

The overall survival (OS) was calculated from the date of the baseline evaluation (initial ABI/ENZ treatment data) to the last follow-up. The OS rates were estimated using the Kaplan-Meier method. A Cox proportional hazards model was used for the univaridate and multivariable analyses. P values of $<$ 0.05 were considered to indicate statistical significance in all statistical tests. The statistical analyses were performed using the SPSS (version 25.0; SPSS Inc., Chicago, IL, USA), R version 3.5.1 (R, Foundation for Statistical Computing, Vienna, Austria) and GraphPad Prism (La Jolla, CA, USA) software programs. 


\section{Nomogram development}

The nomogram for the OS was developed using a Cox proportional hazards regression model with the age, initial PSA, initial stage (M0/M1), Gleason score, time to CRPC, Chemotherapy, PSA at baseline, ALP at baseline, and LDH at baseline as the predictors. Calibration was performed using the methods described by lasonos et al. [10]. The data were randomly separated into training and validation data sets to calibrate the nomogram prediction. The prediction was evaluated by comparing the predicted survival probability at two years with the observed survival probability using the training and validation data sets. The nomogram was also assessed for discriminatory ability with the concordance index (C-index). We repeated 5 -fold cross-validation 2000 times to estimate the $\mathrm{C}$-index and reported the means of the estimated $\mathrm{C}$-index using the training and validation sets.

\section{Results}

The median (range) observational period was $13.3(0.2-52.2)$ months. A total of 189 of the 589 patients (33.2\%) died, and the median OS was 24.7 months [Fig. 1]. The patients' characteristics are summarized in Table 1. A multivariable analysis showed that the time to CRPC, pre-chemotherapy, baseline PSA, baseline ALP, and baseline LDH were independent risk factors for the OS (time to CRPC: hazard ratio [HR] $=0.521,95 \%$ confidence interval $[\mathrm{Cl}]=0.349-0.776, \mathrm{p}=0.001$, pre-chemotherapy: $\mathrm{HR}=1.683,95 \% \mathrm{Cl}=$ 1.232-2.300, $p=0.001$, baseline PSA: $\mathrm{HR}=01.439,95 \% \mathrm{Cl}=1.179-1.755, \mathrm{p}<0.001$, baseline $\mathrm{ALP}=\mathrm{HR}=$ $1.827,95 \% \mathrm{Cl}=1.102-3.028, \mathrm{p}=0.019$, baseline LDH: $\mathrm{HR}=12.123,95 \% \mathrm{Cl}=5.343-27.51, \mathrm{p}<0.001)$ [Table 2]. 
Table 2

Univariate and multivariate analysis to predict prognosis

Univariate analysis

\begin{tabular}{|c|c|c|c|c|c|c|c|c|}
\hline & \multirow{2}{*}{$\begin{array}{l}\mathrm{p} \\
\text { value }\end{array}$} & \multirow[t]{2}{*}{$\mathrm{HR}$} & \multicolumn{2}{|c|}{$95.0 \% \mathrm{Cl}$} & \multirow{2}{*}{$\begin{array}{l}\mathrm{p} \\
\text { value }\end{array}$} & \multirow[t]{2}{*}{$\mathrm{HR}$} & \multicolumn{2}{|c|}{$95.0 \% \mathrm{Cl}$} \\
\hline & & & Lower & Upper & & & Lower & Upper \\
\hline Initial PSA & 0.820 & 1.020 & 0.863 & 1.204 & 0.157 & 0.862 & 0.701 & 1.059 \\
\hline Initial stage M0 vs M1 & 0.091 & 0.776 & 0.578 & 1.042 & 0.469 & 0.876 & 0.611 & 1.254 \\
\hline $\begin{array}{l}\text { Gleason score } 8-10 \text { vs } \\
6-7\end{array}$ & 0.848 & 1.034 & 0.737 & 1.450 & 0.550 & 0.897 & 0.629 & 1.280 \\
\hline Time to CRPC & $<0.001$ & 0.434 & 0.304 & 0.621 & 0.001 & 0.521 & 0.349 & 0.776 \\
\hline $\begin{array}{l}\text { Chemotherapy Yes vs } \\
\text { No }\end{array}$ & $<0.001$ & 2.300 & 1.731 & 3.057 & 0.001 & 1.683 & 1.232 & 2.300 \\
\hline Age at baseline & 0.600 & 0.995 & 0.975 & 1.015 & 0.250 & 1.012 & 0.992 & 1.032 \\
\hline PSA at baseline & $<001$ & 1.958 & 1.680 & 2.282 & $<.001$ & 1.439 & 1.179 & 1.755 \\
\hline ALP at baseline & $<0.001$ & 5.138 & 3.509 & 7.524 & 0.019 & 1.827 & 1.102 & 3.028 \\
\hline LDH at baseline & $<0.001$ & 45.886 & 23.15 & 90.953 & $<.001$ & 12.123 & 5.343 & 27.51 \\
\hline
\end{tabular}

PSA: Prostate-specific antigen, ALP: alkaline phosphatase, LDH: lactate dehydrogenase, CRPC:

Castration-resistant prostate cancer

Figure 2 shows the nomogram for predicting the one- and two-year survival using five statically significant risk factors (time to CRPC, pre-chemotherapy, baseline PSA, baseline ALP, and baseline LDH) and four clinically important risk factors (age, initial PSA, initial metastatic status, and Gleason score). Figures $3 \mathrm{a}$ and $3 \mathrm{~b}$ show the calibrations of the nomogram for the two-year survival. The blue diagonal line indicated the ideal reference line at which predicted the probabilities match the observed proportions. The vertical lines across the blue line represent the nomogram-predicted probabilities grouped for each of the four quartile groups, along with the respective $95 \%$ confidence intervals. From Fig. 3a, we can see the predicted survival rate from the nomogram was well correlated with the actual observation of the twoyear survival in the training data set. Figure $3 b$ shows the calibration of the nomogram for the two-year survival using randomly selected validation data set. We can also see that the predicted survival rate from the nomogram was well-correlated with the actual observation of the two-year survival in the validation set. The means of estimated c-index were 0.72 for training data sets and 0.71 for validation data sets in the 5-fold cross-validations.

We also developed app to easily use these findings in daily clinical practice [Fig. 4] The app were developed for the Android and iOS systems [Fig. 5]. 


\section{Discussion}

This study developed and validated a nomogram for predicting the prognosis of mCRPC patients who receive $\mathrm{ABI}$ and/or ENZ. This nomogram used the initial PSA, initial metastasis status, Gleason score, time to CRPC, previous use of docetaxel or not, age at ABI/ENZ installation, and laboratory data, including the PSA/ALP/LDH at the time of ABI/ENZ installation. The prognosis of mCRPC varies among metastatic lesions. Regarding non-metastatic CRPC (mOCRPC), the PROSPER, SPARTAN, and ARAMIS studies showed the median radiographic progression-free survival (rPFS) to be around 36.6 to 40.4 months [1113]. Although the final OS was not reached, the OS was expected to be around 67.0 to 73.9 in both groups [14]. Regarding metastatic pre-docetaxel chemotherapy CRPC, the PREVAIL or COU-AA-302 studies showed that the OS was around 32.4 to 34.7 months $[1,15]$. Furthermore, regarding metastatic postdocetaxel chemotherapy CRPC, the AFFIRM and COUA-AA-301 showed that the OS was around 14.8 to 18.4 months $[2,16]$. Finally, in real-world metastatic CRPC, the OS was found to be 31.6 months in cases of lymph-node metastasis, 21.3 months in cases of bone metastasis, 19.4 months in cases of lung metastasis, and 13.5 months in cases of liver metastasis [17]. While the OS was speculated in metastatic site, the detailed prognostic estimation using multiple risk factors has not been established. To make right treatment decisions for the right patient at right timing, a detailed prognosis estimation is needed. Recent studies have shown that elevated tumor markers, such as LDH and ALP, and some inflammatory markers are poor prognostic factors for CRPC [6]. Zhao et al. developed a prognostic nomogram for CRPC among Chinese patients using a 449-patient cohort [18]. This nomogram used the Gleason score, presence of intraductal carcinoma of the prostate, baseline ALP/PSA/Hb, and the Eastern Cooperative Oncology Group performance status. Yang et al. also developed a nomogram using the presence of liver metastasis, hemoglobin level, and time from initial ADT to ABI in 110 Chinese CRPC patients [19]. Lin et al. developed a nomogram using the PSA-doubling time, time to PSA progression, and presence of pain in 167 Chinese CRPC patients [20]. This is the first study to predict the prognosis in mCRPC patients in a total of 589 patients who received $A B I / E N Z$.

In mCRPC treatment, newly established medications are more expensive than ADT or docetaxel treatment, which eventually affects a country's insurance system $[6,21]$. Previous studies also created nomograms to predict the prognosis in Chinese CRPC patients [ref]. The economic range in Asia is vast, so nomograms specific to each country are needed. Under the Japanese medical insurance system, all patients received government-approved CRPC medication, including ABI/ENZ, Ra-223, docetaxel, and cabazitaxel. Speleucel $T$ is not approved in Japan. The present findings are expected to benefit Japanese mCRPC patients who are introduced to ABI/ENZ.

Several limitations associated with the present study warrant mention. First, this study used a retrospective cohort divided into a training group and control group from Japanese multicenter hospitals. 
Most of the hospitals were third referral cancer centers. Thus, a further study is needed to confirm the accuracy of these findings using all hospitals, including private clinics. Second, other mCRPC treatment drugs, such as PARP inhibitors and immune-checkpoint inhibitors, will be available in the near future. Once these new treatments are approved, additional validation will likely be needed.

In conclusion, we developed and validated a nomogram for predicting the prognosis of mCRPC patients who receive $A B I / E N Z$ treatment using clinical information and also developed app to facilitate clinical usage.

\section{Conclusion}

We developed nomograms to predict the OS for Japanese MCRPC patients who received ABI and/or ENZ. This nomogram might help clinicians make treatment decisions.

\section{Declarations}

\section{Competing interests}

The authors declare that they have no competing interests.

\section{Authors' Contributions}

Conception and design: TK, YM. Developed and validated the nomogram: YS, Acquisition of data: TK, YS, SY, MK, IK, HY, OK, KT, HT, MI, MY, HU, YM. Drafting of the manuscript: TK, YM. All authors have read and approved the manuscript

\section{Availability of supporting data}

Due to ethical restrictions, the raw data underlying this paper are available upon request to the corresponding author.

\section{FUNDING DECLARATION}

We obtained no funding for this study

\section{ACKNOWLEDGEMENT}

Nomogram app was programmed by Shun Omatsu (Kameciti Create, Tokyo, Japan)

\section{References}


1. Ryan CJ, Smith MR, de Bono JS, Molina A, Logothetis CJ, de Souza P, Fizazi K, Mainwaring P, Piulats $\mathrm{JM}, \mathrm{Ng} \mathrm{S}$, et al. Abiraterone in metastatic prostate cancer without previous chemotherapy. $\mathrm{N}$ Engl J Med. 2013;368(2):138-48.

2. Scher HI, Fizazi K, Saad F, Taplin ME, Sternberg CN, Miller K, de Wit R, Mulders P, Chi KN, Shore ND, et al. Increased survival with enzalutamide in prostate cancer after chemotherapy. N Engl J Med. 2012;367(13):1187-97.

3. Ninomiya S, Kawahara T, Tatenuma T, Miyoshi Y, Miyamoto H, Yao M, Uemura H. Preference for enzalutamide capsules versus tablet pills in patients with prostate cancer. Int J Urol. 2019;26(12):1161-2.

4. Bilusic M, Madan RA, Gulley JL. Immunotherapy of Prostate Cancer: Facts and Hopes. Clin Cancer Res. 2017;23(22):6764-70.

5. Ku SY, Gleave ME, Beltran H. Towards precision oncology in advanced prostate cancer. Nat Rev Urol. 2019;16(11):645-54.

6. Ohno Y. Role of systemic inflammatory response markers in urological malignancy. Int J Urol. 2019;26(1):31-47.

7. Partin AW, Yoo J, Carter HB, Pearson JD, Chan DW, Epstein JI, Walsh PC. The use of prostate specific antigen, clinical stage and Gleason score to predict pathological stage in men with localized prostate cancer. J Urol. 1993;150(1):110-4.

8. Shariat SF, Karakiewicz PI, Suardi N, Kattan MW. Comparison of nomograms with other methods for predicting outcomes in prostate cancer: a critical analysis of the literature. Clin Cancer Res. 2008;14(14):4400-7.

9. Spreafico A, Chi KN, Sridhar SS, Smith DC, Carducci MA, Kavsak P, Wong TS, Wang L, Ivy SP, Mukherjee SD, et al. A randomized phase II study of cediranib alone versus cediranib in combination with dasatinib in docetaxel resistant, castration resistant prostate cancer patients. Invest New Drugs. 2014;32(5):1005-16.

10. Iasonos A, Keung EZ, Zivanovic O, Mancari R, Peiretti M, Nucci M, George S, Colombo N, Carinelli S, Hensley $\mathrm{ML}$, et al. External validation of a prognostic nomogram for overall survival in women with uterine leiomyosarcoma. Cancer. 2013;119(10):1816-22.

11. Hussain M, Fizazi K, Saad F, Rathenborg P, Shore N, Ferreira U, Ivashchenko P, Demirhan E, Modelska $\mathrm{K}$, Phung, et al. Enzalutamide in Men with Nonmetastatic, Castration-Resistant Prostate Cancer. N Engl J Med. 2018;378(26):2465-74.

12. Smith MR, Saad F, Chowdhury S, Oudard S, Hadaschik BA, Graff JN, Olmos D, Mainwaring PN, Lee JY, Uemura H, et al. Apalutamide Treatment and Metastasis-free Survival in Prostate Cancer. N Engl J Med. 2018;378(15):1408-18.

13. Fizazi K, Shore N, Tammela TL, Ulys A, Vjaters E, Polyakov S, Jievaltas M, Luz M, Alekseev B, Kuss I, et al. Darolutamide in Nonmetastatic, Castration-Resistant Prostate Cancer. N Engl J Med. 2019;380(13):1235-46.

14. ASC02020: https://meetings.asco.org/am/virtual-welcome. ASCO2020 Abstract 2020. 
15. Beer TM, Armstrong AJ, Rathkopf DE, Loriot Y, Sternberg CN, Higano CS, Iversen P, Bhattacharya S, Carles J, Chowdhury S, et al. Enzalutamide in metastatic prostate cancer before chemotherapy. $\mathrm{N}$ Engl J Med. 2014;371(5):424-33.

16. de Bono JS, Logothetis CJ, Molina A, Fizazi K, North S, Chu L, Chi KN, Jones RJ, Goodman OB Jr, Saad $\mathrm{F}$, et al. Abiraterone and increased survival in metastatic prostate cancer. $\mathrm{N}$ Engl $\mathrm{J}$ Med. 2011;364(21):1995-2005.

17. Halabi S, Kelly WK, Ma H, Zhou H, Solomon NC, Fizazi K, Tangen CM, Rosenthal M, Petrylak DP, Hussain $\mathrm{M}$, et al. Meta-Analysis Evaluating the Impact of Site of Metastasis on Overall Survival in Men With Castration-Resistant Prostate Cancer. J Clin Oncol. 2016;34(14):1652-9.

18. Hanczyc P, Prastowska E, Wieczorek E. [Circulating immune complexes in retinitis pigmentosa]. Klin Oczna. 1988;90(6):203-4.

19. Yang YJ, Lin GW, Li GX, Dai B, Ye DW, Wu JL, Xie HY, Zhu Y. External validation and newly development of a nomogram to predict overall survival of abiraterone-treated, castration-resistant patients with metastatic prostate cancer. Asian J Androl. 2018;20(2):184-8.

20. Lin GW, Ye DW, Jia HX, Dai B, Zhang HL, Zhu Y, Shi GH, Ma CG. Development of a preliminary nomogram to predict progression of bone scan for castration-resistant prostate cancer. Onco Targets Ther. 2015;8:713-9.

21. Afriansyah A, Hamid ARA, Mochtar CA, Umbas R. Survival analysis and development of a prognostic nomogram for bone-metastatic prostate cancer patients: A single-center experience in Indonesia. Int J Urol. 2019;26(1):83-9.

\section{Figures}




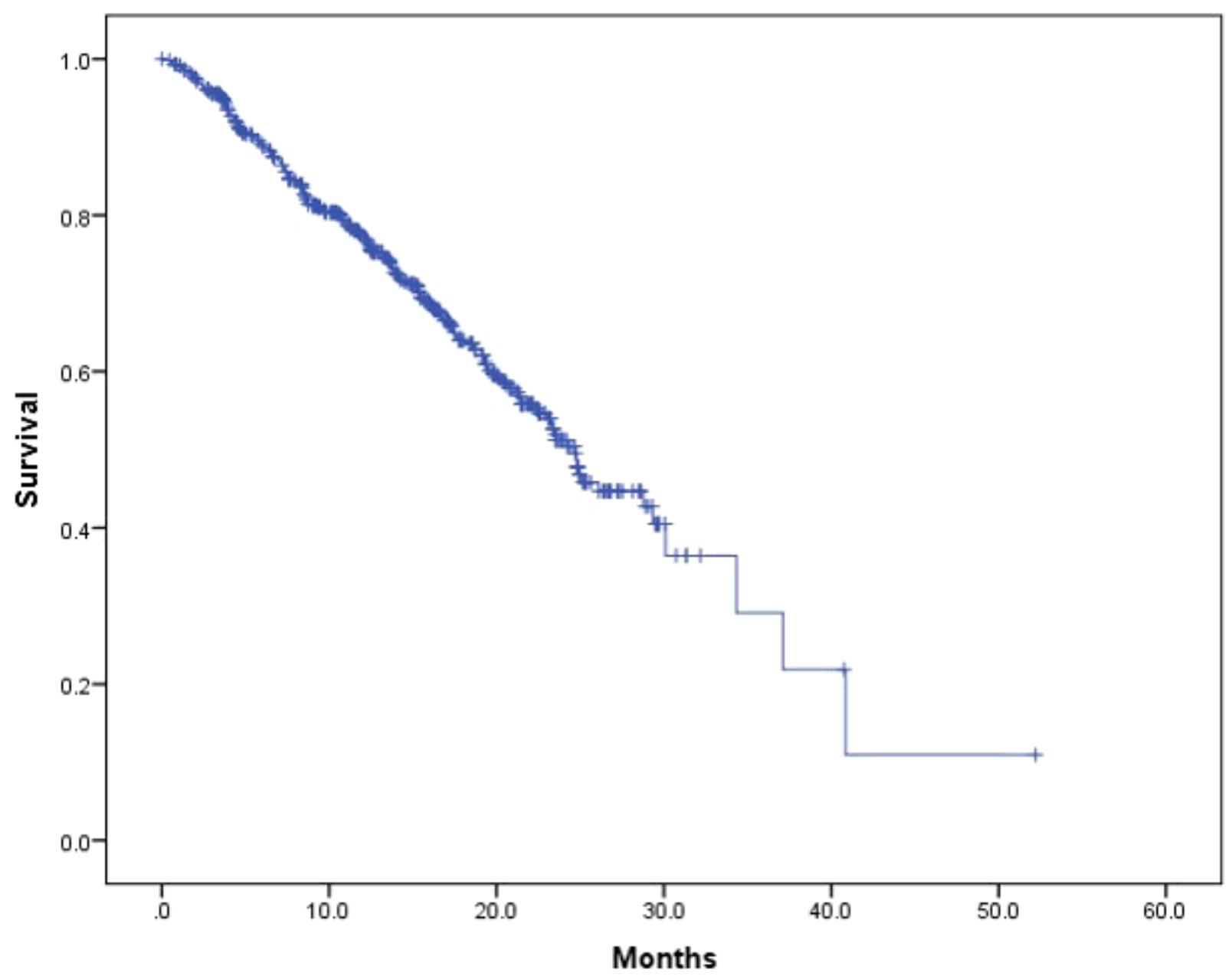

Figure 1

Kaplan-Meier curve for the overall survival in metastatic castration-resistant prostate cancer patients treated with abiraterone and/or enzalutamide 


\begin{abstract}
Initial PSA (ng/mL)
Initial stage (M0 vs M1)

$\vec{\delta} \vec{\delta} \vec{\circ} \vec{\circ} \overrightarrow{0}$

넝
\end{abstract}

Gleason score

$$
\overrightarrow{o l}^{\mathrm{N}}
$$

Time to CRPC (months)

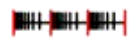

$$
\begin{aligned}
& \overrightarrow{8} \overrightarrow{0} \rightarrow
\end{aligned}
$$

Chemotherapy (No vs Yes)

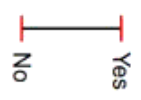

Age at baseline (years)

$$
\underset{\text { ํㅗㅇํㅇㅇㅛ }}{\mathrm{H}}
$$

PSA at baseline $(\mathrm{ng} / \mathrm{mL})$

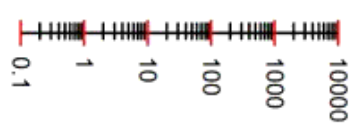

ALP at baseline (IU/L)

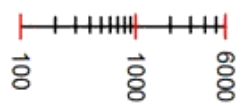

LDH at baseline (IU/L)

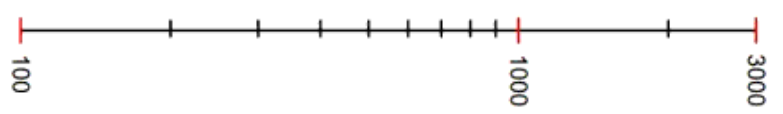

Points

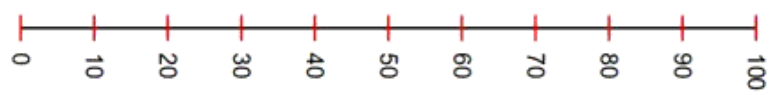

Total points

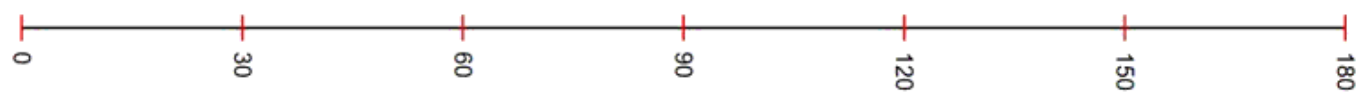

1 year survival

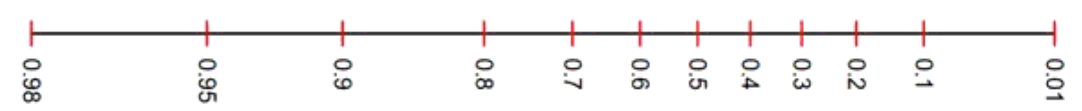

2 year survival

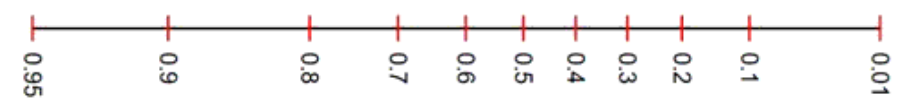

\title{
Figure 2
}

Nomogram for predicting the one- and two-year survival among men treated with abiraterone acetate and/or enzalutamide for metastatic castration-resistant prostate cancer. 
a)

\section{Training cohort}

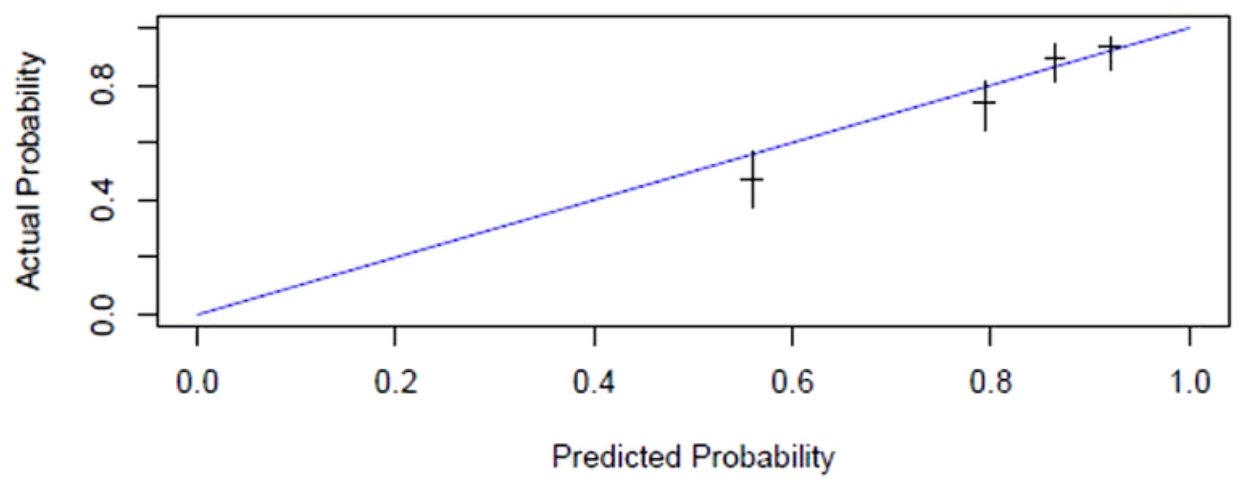

\section{Validation cohort}

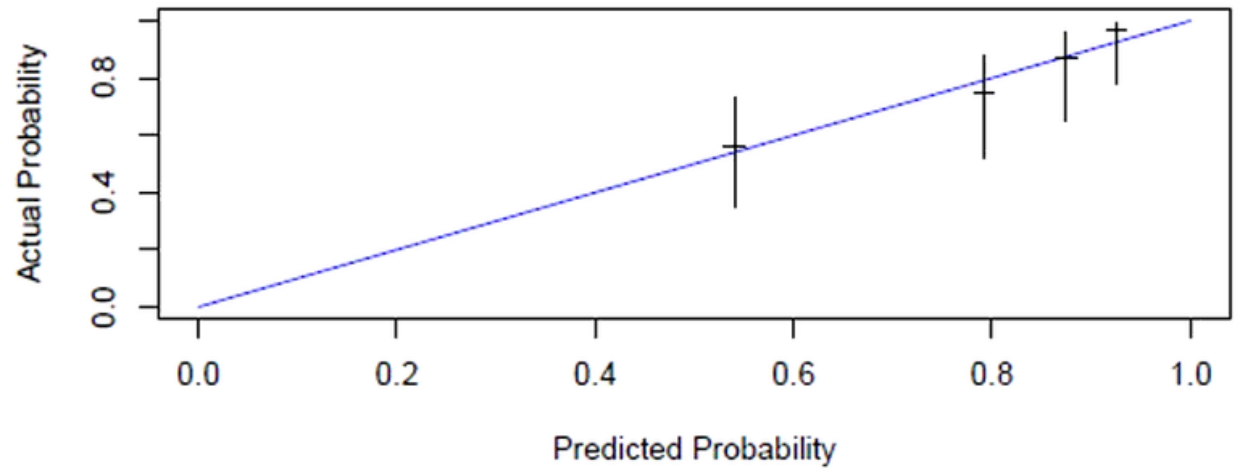

Figure 3

Calibration plots of the nomogram for the two-year survival. a) for training cohort and b) for validation cohort.
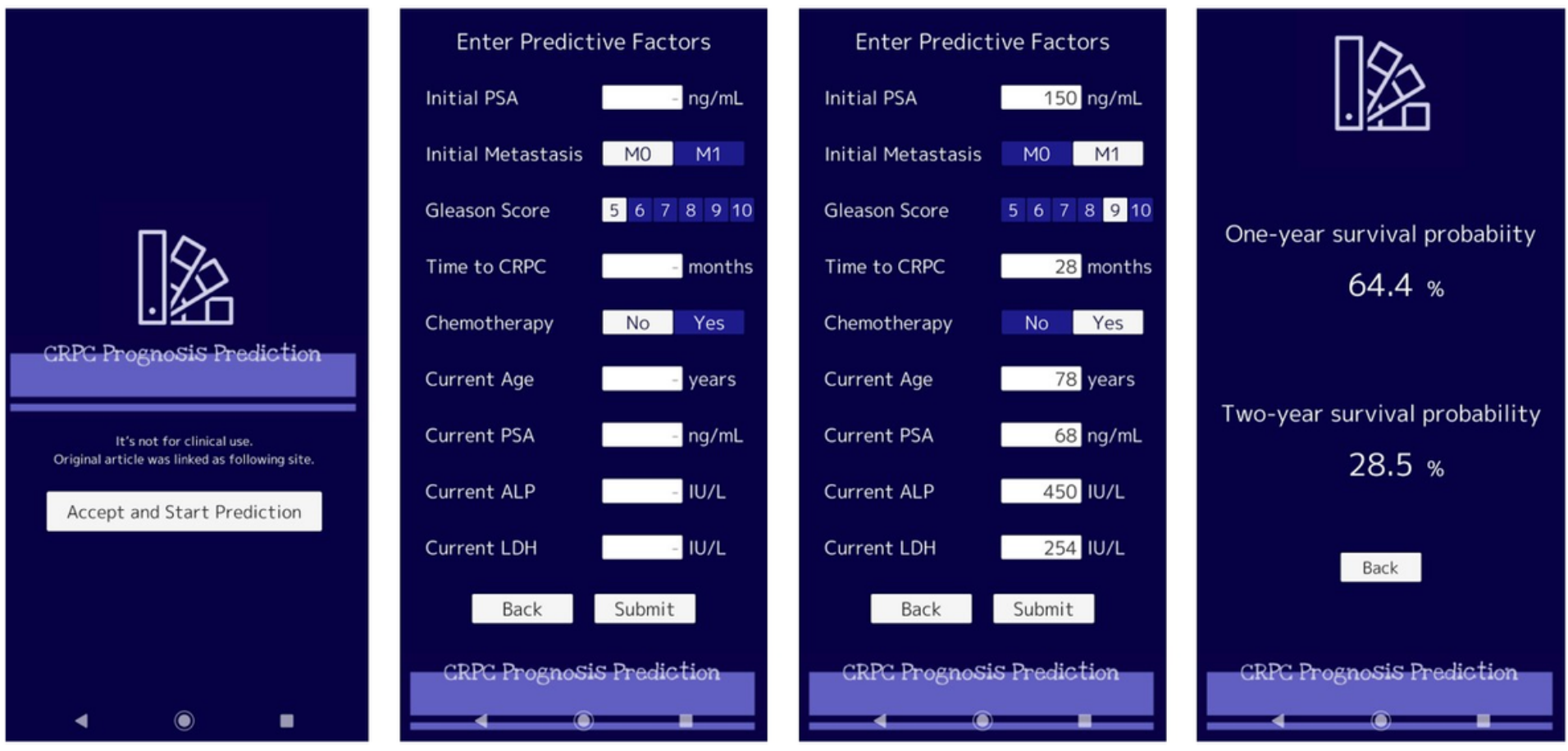
Figure 4

Android and iphone/iOS app to predict prognosis for 1-year and 2-year survival among men treated with abiraterone acetate and/or enzalutamide for metastatic castration-resistant prostate cancer.

a)

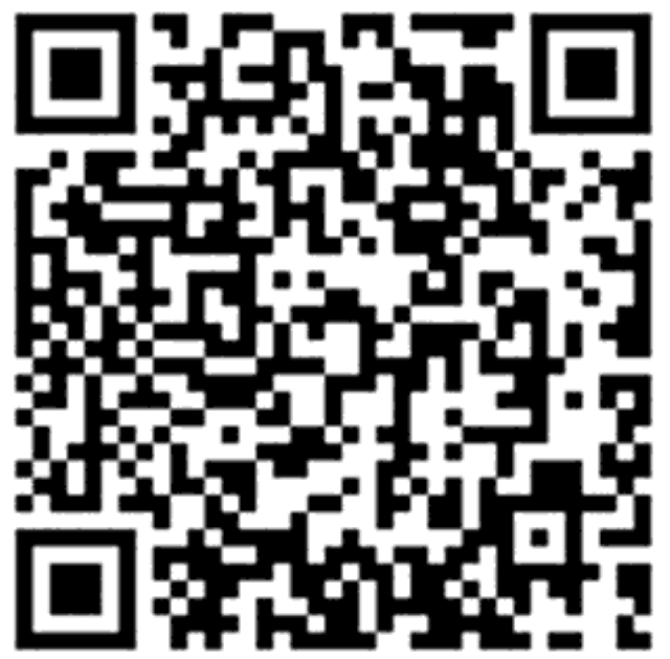

b)
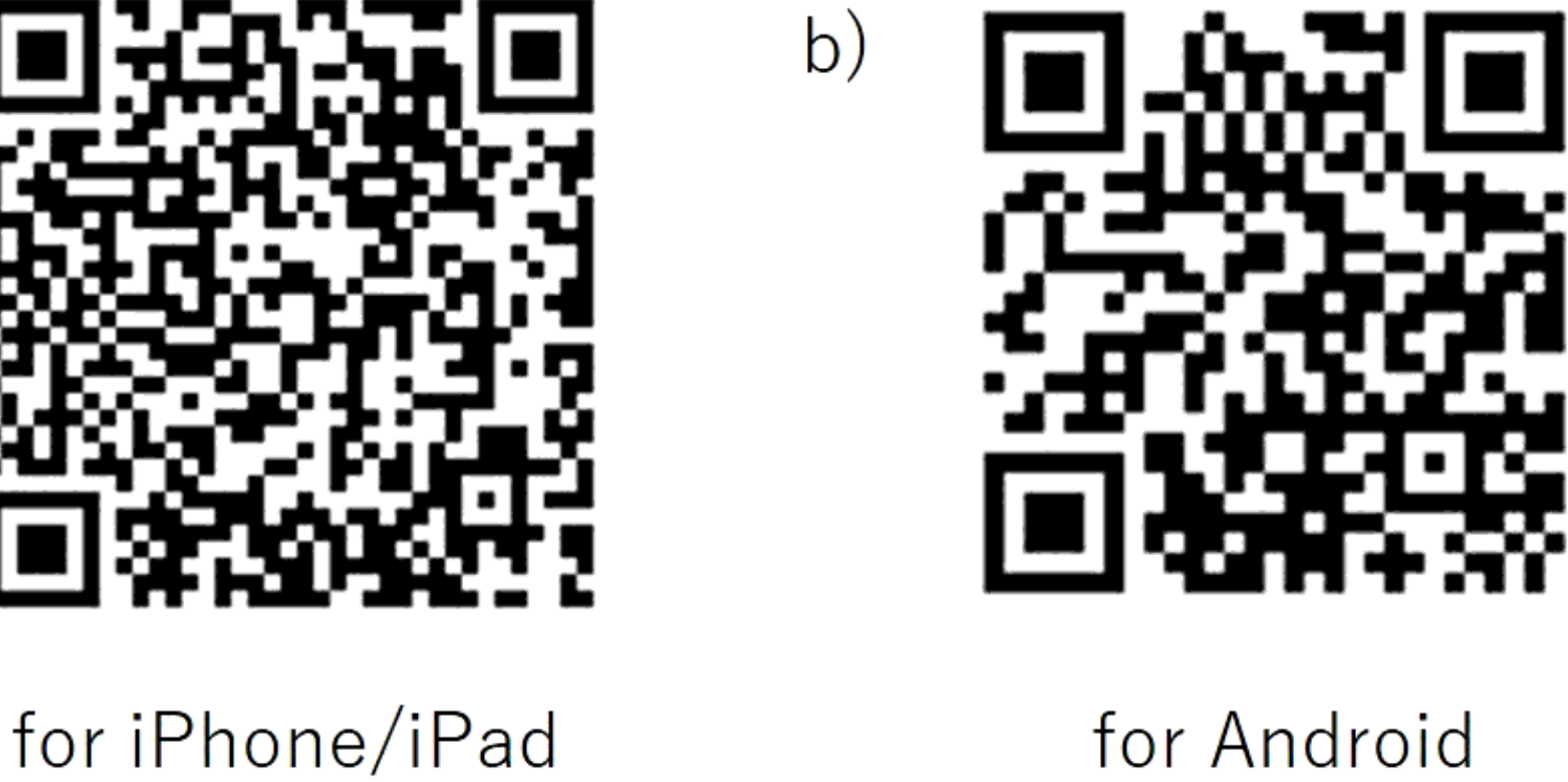

for Android

Figure 5

QR code to download the a) Android and b) iPhone/iPad app. 